\title{
CONTINUED FRACTIONS AND EQUIVALENT COMPLEX NUMBERS
}

\author{
RICHARD B. LAKEIN
}

\begin{abstract}
In this note it is shown, by a counterexample, that the familiar theorem on the continued fraction expansions of equivalent numbers does not hold when these notions are extended to complex numbers.
\end{abstract}

Two real numbers $x, x^{\prime}$ are called equivalent, $x \sim x^{\prime}$, if

(1) $x^{\prime}=(a x+b) /(c x+d)$ for some $a, b, c, d \in Z, a d-b c= \pm 1$.

Consider also the continued fraction (CF) expansion of a real number

$$
x=\left(a_{0}, a_{1}, \cdots, a_{n-1}, x_{n}\right), \quad x_{n-1}=a_{n-1}+1 / x_{n},
$$

where $x_{n}$ is the $n$th complete quotient of $x$. It is a standard theorem in CF's that $x \sim x^{\prime}$ if and only if, in the CF expansions of $x$ and $x^{\prime}$, there exist $m, n$ such that $a_{m+k}=a_{n+k}^{\prime}$ for all $k \geqq 0$-more briefly, $x_{m}=x_{n}^{\prime}$.

Hurwitz, in a paper [2] on the "nearest integer" CF (where the partial quotients $a_{n}$ may be negative) proved that essentially the same result carries over. That is, $x \sim x^{\prime}$ if and only if there exist $m, n$ such that $x_{m}=$ $\pm x_{n}^{\prime}$, where these are complete quotients of the nearest integer CF's. Hurwitz also defined [1] a complex generalization of the nearest integer CF (it might be called the "nearest Gaussian integer" CF) by which a complex number $x$ is expanded in a simple CF as in (2) with partial quotients $a_{n}$ in $Z[i]$. There is an analogous notion of equivalent numbers as in (1), where $a, b, c, d \in Z[i], a d-b c= \pm 1, \pm i$.

Although this complex CF has many analogies to real CF's, the expected theorem on equivalent numbers fails, as shown by a

COUNTEREXAMPLE. Let $\Omega=\frac{1}{2}\left(i+(43+28 i)^{1 / 2}\right), \quad A=(5-i+\Omega) /(4-i)$, $B=(3+2 i+\Omega) / 4$. Then $A \sim B$, in fact $A=(2 B-i) /(B-i)$. However the CF expansions of $A$ and $B$, which are periodic, are distinct:

$$
\begin{aligned}
& A=\overline{(2+i, 3 i,-1+2 i,-1+2 i, 3,-2-i)}, \\
& B=\overline{(2+i,-2+i,-2+i, 1-2 i,-1-2 i, 1+2 i)} .
\end{aligned}
$$

Thus $A_{m}= \pm B_{n}$, or even $\pm i B_{n}$, never holds.

Received by the editors May 8, 1973.

AMS (MOS) subject classifications (1970). Primary 10F20; Secondary 12A05.

Key words and phrases. Complex continued fractions, equivalent complex numbers.

(c) American Mathematical Society 1974 
Finally we note that an incomplete partial result may hold for a different, less natural type of complex CF. See [3], [4, p. 88].

\section{REFERENCES}

1. A. Hurwitz, Über die Entwicklung komplexer Grössen in Kettenbrüche, Acta Math. 11 (1888), 187-200 (= Werke II, 72-83).

2. — Über eine besondere Art der Kettenbruch-Entwicklung reeller Grössen, Acta Math. 12 (1889), 367-405. (= Werke II, 84-115).

3. J. Hurwitz, Uber die Reduction der binären quadratischen Formen mit complexen Coefficienten und Variabeln, Acta Math. 25 (1902), 231-290.

4. A. Stein, Die Gewinnung der Einheiten in gewissen relativ-quadratischen Zahlkörpern durch das J. Hurwitzsche Kettenbruchverfahren, J. Reine Angew. Math. 156 (1927), 69-92.

Department of Mathematics, State Universtiy of New York at Buffalo, AMHERST, NEW YORK 14226 